\title{
Antitumor activity of Chidamide in hepatocellular carcinoma cell lines
}

\author{
HAIJUAN WANG $^{1 *}$, YI GUO ${ }^{2 *}$, MING FU $^{1}$, XIAO LIANG ${ }^{1}$, XUEYAN ZHANG ${ }^{1}$, \\ RENZHI WANG ${ }^{2}$, CHEN LIN $^{1}$ and HAILI QIAN ${ }^{1}$ \\ ${ }^{1}$ State Key Laboratory of Molecular Oncology, Cancer Institute/Hospital, CAMS and PUMC; \\ ${ }^{2}$ Department of Neurosurgery, Peking Union Hospital, Beijing, P.R. China
}

Received December 13, 2011; Accepted March 29, 2012

DOI: $10.3892 / \mathrm{mmr} .2012 .858$

\begin{abstract}
Chidamide, the structural analog of MS-275, is a novel and promising histone deacetylase (HDAC) inhibitor for use in cancer therapy. To investigate its effects on cancer cell growth, MTT assay was performed in 10 human cancer cell lines. The data showed that the $\mathrm{IC}_{50}$ of Chidamide ranged from 1 to $13 \mu \mathrm{M}$, which was comparable to that of MS-275 in half of the tested cell lines. Furthermore, the growth curve indicated that cell growth was gradually inhibited with an increase in Chidamide dosage, and the inhibition was reversed after drug removal in two hepatocelluclar carcinoma cell lines, BEL-7402 and HCC-9204. To determine cell cycle and apoptosis, FACS was carried out in the BEL-7402 and HCC-9204 cells treated with Chidamide. A decrease in the cell population at $\mathrm{S}$ phase and an increase in the cell population at G1 phase occurred in a dose-dependent manner. In addition, Chidamide induced apoptosis and up-regulated p21 mRNA expression. These results suggest that Chidamide may arrest the cell cycle and inhibit the growth of hepatocellular carcinoma cells through up-regulation of p21. Further studies are required to clarify the antitumor activity of Chidamide in vivo and its mechanism in anticancer therapy.
\end{abstract}

\section{Introduction}

Histone acetylases (HATs) and histone deacetylases (HDACs) are a series of enzymes functioning to acetylate and deacetylate the amino-terminal lysine residues of histones, which result in the remodeling of the chromatin structures and affect the accessibility of the chromatin to transcription factors to start gene transcription. It has been known that many tumors are

Correspondence to: Dr Haili Qian, State Key Laboratory of Molecular Oncology, Cancer Institute/Hospital, CAMS and PUMC, Rm9-7, Research Building, 17 Panjiayuan Nanli, Chaoyang, Beijing 100021, P.R. China

E-mail: qianhaili001@163.com

*Contributed equally

Key words: Chidamide, antitumor, p21, apoptosis characterized by an imbalance of HDAC and HAT activity (1). HDACs regulate the expression of genes regulating a broad range of cell functions, such as tumor suppression, cell cycle, differentiation and DNA repair. Hence, they are considered as an important class of targets in cancer treatment strategies, and HDAC inhibitors are becoming a new promising class of anticancer drugs (2).

To date, four classes of HDACs have been identified, which have been grouped into three families (1). Class I and II are inhibited by trichostatin A (TSA); class III HDACs are a family of $\mathrm{NAD}^{+}$-dependent proteins and are not sensitive to TSA; class IV has only one member, HDAC11, whose structure is homologous to class I and II. Recently, HDAC inhibitors, including TSA, sodium butyrate, suberoylanilide hydroxamic acid, depsipeptide and MS-275, are already in pre-clinical and clinical development (3-6). Although they are structurally diverse compounds, they share the potential to prevent cell cycle progression in the G1 and G2/M phases, leading to growth arrest, differentiation and/or death of cancer cells. The precise mechanism underlying cell cycle arrest or differentiation through histone acetylation is unknown, but definitely, the effect of most HDAC inhibitors on G1 arrest is closely related to the activated transcription of the cyclin-dependent kinase inhibitor $\mathrm{p} 21^{\mathrm{CIP} 1 / \mathrm{WAF}}$.

To develop more candidate HDAC inhibitors for evaluation in cancer therapy, herein we present a novel HDAC inhibitor, $\mathrm{N}$-(2-amino-5-fluorine benzyl)-4-[N-(pyridine-3-acrylyl) ammonia methyl] benzamide (Chidamide), which is structurally homologous to MS-275, to explore its antitumor functions in a variety of cancer cells. The data showed that Chidamide treatment inhibits the proliferation of a series of cancer cell lines. Moreover, treatment of hepatocellular cancer cells with Chidamide induced cell cycle arrest and apoptosis along with up-regulation of p21 expression.

\section{Materials and methods}

Regents. Chidamide (N-(2-amino-5-fluorophenyl)-4-[N(pyridin-3-ylacryloyl) aminomethyl] benzamide) and MS-275 (N-(2-aminophenyl)-4-[N-(pyridin-3-ylmethoxycarbonyl) aminomethyl] benzamide) were kindly provided by Shenzhen Chipscreen Biosciences Ltd. (Shenzhen, China), dissolved in dimethyl sulfoxide (DMSO) at a concentration of $20 \mathrm{mM}$ 
Table I. Effect of Chidamide and MS-275 on growth inhibition of human cancer cell lines.

\begin{tabular}{|c|c|c|c|c|c|}
\hline \multirow[t]{2}{*}{ Cell line } & \multirow[t]{2}{*}{ Origin } & \multicolumn{2}{|c|}{$\mathrm{IC}_{50}(\mu \mathrm{M})$} & \multirow[t]{2}{*}{ P-value } & \multirow[t]{2}{*}{ p53 status } \\
\hline & & Chidamide & MS-275 & & \\
\hline BEL-7402 & Liver & $5.88 \pm 1.01$ & $1.55 \pm 0.221$ & $<0.01$ & wt \\
\hline HCC-9204 & Liver & $12.8 \pm 2.05$ & $15.50 \pm 3.550$ & $>0.05$ & $\mathrm{mt}$ \\
\hline A549 & Lung & $3.88 \pm 1.46$ & $2.54 \pm 0.334$ & $>0.05$ & wt \\
\hline 973 & Lung & $6.77 \pm 1.76$ & $2.79 \pm 0.831$ & $>0.05$ & $\mathrm{mt}$ \\
\hline MCF-7 & Breast & $2.47 \pm 0.722$ & $2.03 \pm 0.235$ & $>0.05$ & wt \\
\hline MDA-MB-231 & Breast & $1.37 \pm 0.101$ & $1.69 \pm 0.237$ & $>0.05$ & $\mathrm{mt}$ \\
\hline EC-109 & Esophageal & $10.6 \pm 2.09$ & $4.55 \pm 1.250$ & $<0.05$ & un \\
\hline EC-9706 & Esophageal & $12.8 \pm 2.05$ & $2.25 \pm 0.436$ & $<0.01$ & un \\
\hline HCT-8 & Colon & $7.25 \pm 1.04$ & $1.20 \pm 0.326$ & $<0.01$ & wt \\
\hline SW1990 & Pancreas & $4.55 \pm 1.25$ & $0.774 \pm 0.206$ & $<0.05$ & wt \\
\hline
\end{tabular}

Tumor cells were exposed to Chidamide and MS-275 for $72 \mathrm{~h}$, after which the cell medium was removed and cells were treated with MTT for $4 \mathrm{~h}$. Then, the absorbance was detected at $490 \mathrm{~nm}$. IC $\mathrm{I}_{50}$ was determined by SPSS software. wt, wild-type; mt, mutated; un, unknown.

and then kept at $-20^{\circ} \mathrm{C}$ until use. Methylthiazolyl tetrazolium (MTT) was purchased from Sigma (St. Louis, MO, USA).

Cell lines and culture. The BEL-7402 and HCC-9204 human hepatocellular cancer cell lines, the 973 human lung cancer cell line, the MCF-7 and MDA-MB-231 human breast cancer cell lines, the HCT- 8 human colon cancer cell line, and the EC-109 and EC-9706 human esophageal cancer cell lines were obtained from our laboratory. The A549 human lung cancer cell line was kindly provided by Professor Xuyi Liu, Beijing Cancer Research Institute. The SW1990 human pancreas cancer cell line was a gift provided by Dr Shikai Wu, Chinese Academy of Military Medical Sciences. All the cell lines were grown in RPMI-1640 medium supplemented with $10 \%$ fetal calf serum, penicillin and streptomycin in a $37^{\circ} \mathrm{C}$ incubator maintained at $5 \% \mathrm{CO}_{2}$.

Cell viability assay. Cells (5,000-8,000) were plated into each well of 96-well plates and grown overnight. The next day, Chidamide or MS-275 was added at demanded final concentration in triplicate, and the cells were cultivated for an additional 3 days or indicated time-points. After the cell medium was removed, $100 \mu \mathrm{l}$ of MTT solution $(0.5 \mathrm{mg} / \mathrm{ml})$ diluted with culture medium free of serum was added into each well of the plates, and the cells were incubated for $4 \mathrm{~h}$. Supernatant was removed and $100 \mu \mathrm{l}$ DMSO per well was added to the 96-well plates to dissolve the MTT crystals. The plate was read with a microplate reader (Bio-Rad Laboratories, Hercules, CA, USA) at a wavelength of $490 \mathrm{~nm}$. The survival rate of cell proliferation was determined by the equation $\left(\mathrm{OD}_{\text {treated }}-\mathrm{OD}_{\text {blank }}\right) /\left(\mathrm{OD}_{\text {control }}\right.$ $\left.\mathrm{OD}_{\text {blank }}\right) \times 100 \%$. OD is the optical value from the reader. The experiments were independently repeated four times.

Cell cycle analysis. The hepatocellular carcinoma cells were grown and treated with Chidamide or MS-275 in cell culture dishes for indicated time periods. At the end of the treatment, the cells were washed with phosphate-buffered saline (PBS) and fixed with $70 \%$ ethanol overnight at $-20^{\circ} \mathrm{C}$. The following day, the cells were washed again with PBS and treated with RNaseA (at a final concentration of $200 \mu \mathrm{g} / \mathrm{ml}$ ) for $30 \mathrm{~min}$. The cells were washed and resuspended in $0.5 \mathrm{ml} \mathrm{PBS}$ for propidium iodide (PI) incubation at a final concentration of $100 \mu \mathrm{g} / \mathrm{ml}$ for $1 \mathrm{~h}$. The cells were analyzed with the FACS Vantage SE (BD Corporation, Franklin Lakes, NJ, USA), and Cell Quest software version 2.0 (BD Corporation) was used for data analysis.

Reverse transcription-polymerase chain reaction ( $R T-P C R)$. The appropriately treated BEL-7402 and HCC-9204 cells were collected and resuspended in lysis buffer for RNA extraction. RT was conducted using oligo(dT) M-MLV (Moloney murine leukemia virus reverse transcriptase) according to the manufacturer's instructions. Polymerase chain reaction was carried out with a 2400 PCR machine (Perkin Elmer). The parameters for the thermal cycling of PCR were $5 \mathrm{~min}$ at $95^{\circ} \mathrm{C}$ for 1 cycle, $45 \mathrm{sec}$ at $94^{\circ} \mathrm{C}, 45 \mathrm{sec}$ at $55^{\circ} \mathrm{C}, 45 \mathrm{sec}$ at $72^{\circ} \mathrm{C}$ for 32 cycles and $10 \mathrm{~min}$ at $72^{\circ} \mathrm{C}$ for 1 cycle. $P C R$ primers used in the experiment were as follows: tubulin (F) 5'-CTCATCACAGGCAAGGAAGAT-3' and (R) 5'-TTAAGGTAAGTGTAGGTTGGG-3'; p21 WAF1/CIP1 (F) 5'-GATGTCCGTCAGAACCCATGC-3' and (R) 5'-GGA AGGTAGAGCTTGGGCAGGC-3'.

\section{Results}

Chidamide inhibits cell growth of different cancer cell lines. We performed MTT assay to evaluate the $\mathrm{IC}_{50}$ of Chidamide and MS-275 in ten different cell lines. In brief, the cells were exposed to drugs at different concentrations for $72 \mathrm{~h}$, and cell survival rates were calculated. Chidamide effectively inhibited cell growth of the various cell lines, irrespective of p53 status (Table I). Following comparison of the $\mathrm{IC}_{50}$ values between Chidamide and MS-275, these values were at similar levels in five cell lines, while in the other tested five cell lines, the $\mathrm{IC}_{50}$ value of Chidamide was higher compared to that of MS-275 (Table I). To further investigate the effect of Chidamide on cell proliferation, BEL-7402 and HCC-9204 cells were treated with Chidamide at different concentrations that were a bit 
Table II. Effect of chidamide treatment on the cell doubling time of BEL-7402 and HCC-9204 cells.

\begin{tabular}{llc}
\hline Cell line & \multicolumn{1}{c}{ Treatment } & Doubling time (h) \\
\hline BEL-7402 & Vehicle & 24.8 \\
& Chidamide $(1 \mu \mathrm{M})$ & 29.4 \\
& Chidamide $(5 \mu \mathrm{M})$ & - \\
& Chidamide $(5 \mu \mathrm{M}$ for $24 \mathrm{~h})$ & 35.9 \\
HCC-9204 & Vehicle & 27.8 \\
& Chidamide $(2 \mu \mathrm{M})$ & 36.8 \\
& Chidamide $(10 \mu \mathrm{M})$ & - \\
& Chidamide $(10 \mu \mathrm{M}$ for $24 \mathrm{~h})$ & 36.3 \\
\hline
\end{tabular}

-, cells did not achieve a 2-fold increase in number during the 7 days of observation. BEL-7402 and HCC-9204 cells were treated with either medium or Chidamide at indicated concentrations and time-points, and then subjected to MTT assay. To test the reversibility of the effect of Chidamide, the drug in one of the groups with a high concentration of Chidamide was replaced with medium after $24 \mathrm{~h}$ of treatment. Cell number was determined by trypan blue exclusion, and then a cell growth curve was plotted and the cell doubling time was calculated.

lower than the $\mathrm{IC}_{50}$ value. Chidamide significantly extended the cell doubling rates according to concentration in both cell lines (Table II). Notably, the effect of the growth inhibition was reversible when Chidamide was replaced with medium after $24 \mathrm{~h}$ of treatment (Fig. 1A and B).

Chidamide arrests the cell cycle at G1 phase. To determine the effect of Chidamide on the cell cycle, BEL-7402 and HCC-9204 cells were exposed to Chidamide or MS-275 at a concentration close to the $\mathrm{IC}_{50}$ for $24 \mathrm{~h}$ as indicated. Similar to the effect on the MS-275 cells, FACS data showed that Chidamide significantly reduced the $\mathrm{S}$ phase cell fraction, while inducing a marked increase in the G1 phase cell fraction in both cell lines with different p53 statuses (Fig. 2A and B). Furthermore, different concentrations of Chidamide or MS-275 were used to treat BEL-7402 and HCC-9204 cells for $24 \mathrm{~h}$ to investigate the relationship between drug dosage and cell cycle phases. As shown in Table III, Chidamide significantly altered the number of cells in the phase fractions with an increase in dose (i.e., in BEL-7402 cells, from 34.4 to $7.4 \%$ at $\mathrm{S}$ fraction, and from 34.8 to $52.5 \%$ at $\mathrm{G} 1$ fraction). Finally, to determine the time-course effect of Chidamide on the cell cycle, BEL-7402 cells were treated with $5 \mu \mathrm{M}$ Chidamide for $6,12,24$ and $48 \mathrm{~h}$, followed by FACS analysis (Fig. 2C). Chidamide reduced the $\mathrm{S}$ phase cell fraction starting at $6 \mathrm{~h}$ after treatment. The reduction was observed at 24-h exposure and continued further until $48 \mathrm{~h}$. However, the change in the G1 cell fraction appeared from the $12 \mathrm{~h}$ timepoint, which was more obvious with elapse of time. A similar effect was achieved in the MS-275-treated group (Fig. 2D).

Chidamide induces cell apoptosis. To test the effect of Chidamide on cell apoptosis, FACS analysis was performed in BEL-7402 cells exposed to $5 \mu \mathrm{M}$ Chidamide and HCC-9204 cells exposed to $10 \mu \mathrm{M}$ Chidamide, for 24 and $48 \mathrm{~h}$. As shown in Fig. 3, Chidamide induced intense apoptosis in parallel with the treatment period. In BEL-7402 cells, the apoptosis rate
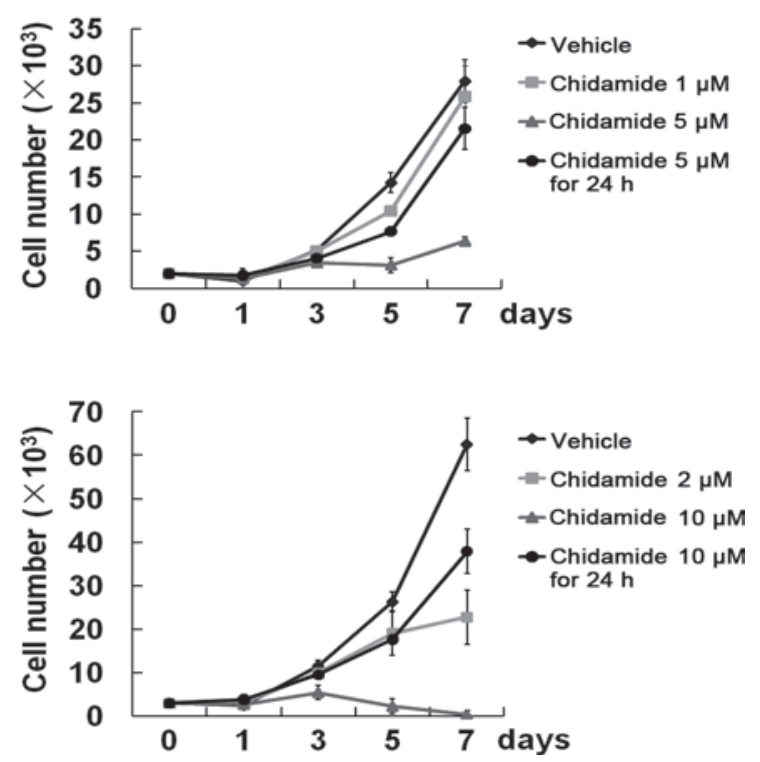

Figure 1. Cell growth curve. (A) BEL-7402 and (B) HCC-9204 cells were exposed to Chidamide at different concentrations. Trypan blue exclusion was used to determine cell number at indicated time-points.

increased by $3.8 \% 24 \mathrm{~h}$ after treatment and by $11.8 \% 48 \mathrm{~h}$ after treatment. These values were 7.8 and $29.8 \%$ in HCC-9204 cells after 24 and $48 \mathrm{~h}$ of treatment, respectively.

Chidamide up-regulates $p 21$ expression in cells with different p53 statuses. $\mathrm{p} 21$ is one of the key players in the process of chemotherapeutic-induced apoptosis in cancer cells. To determine whether cell growth arrest induced by Chidamide was correlated with p21 expression level, which reflects the potential intensity of cell apoptosis, cells were exposed to $5 \mu \mathrm{M}, 10 \mu \mathrm{M}$ Chidamide or MS-275 (BEL-7402), or $10 \mu \mathrm{M}, 20 \mu \mathrm{M}$ Chidamide or MS-275 (HCC-9204) for $48 \mathrm{~h}$. Furthermore, to investigate whether p21 expression was related to time of drug treatment, cells were treated with $5 \mu \mathrm{M}$ (BEL-7402) or $10 \mu \mathrm{M}$ (HCC-9204) Chidamide for 12, 24 or $48 \mathrm{~h}$. As shown in Fig. 4, Chidamide and MS-275 increased p21 mRNA expression with an increase in dose and time. This indicates that Chidamide activates p21 expression independently of $\mathrm{p} 53$, providing advantages to the treatment of cancers with an inactivated p53 status.

\section{Discussion}

HDAC inhibitors suppress the activity of HDAC through binding to HDAC active sites. HDAC inhibitors have been categorized into four classes: hydroxamates, benzamides, short-chain fatty acids and cyclic peptides (4). MS-275 is an artificial benzamide derivative with anticancer effects on various types of cancer, such as lung (7), ovarian (8) and colorectal cancer (9). Chidamide, a structural analog of MS-275, is a novel HDAC inhibitor developed by Chipscreen Biosciences (Shenzhen, China). The drug is currently in NDA-directed clinical studies for relapsed $\mathrm{T}$ cell lymphoma at multi-centers across China and phase I in the US as well. Compared to MS-275, Chidamide is more stable and can be stored at room temperature. Moreover, Chidamide has a longer half-life and is less toxic in vivo than MS-275 (10). 
Table III. Alteration in the cell cycle of BEL-7402 and HCC-9204 cells after exposure to different doses of Chidamide and MS-275 for $24 \mathrm{~h}$.

\begin{tabular}{llccc}
\hline Cell lines & \multicolumn{1}{c}{ Treatment } & G0/G1 phase $(\%)$ & S phase $(\%)$ & G2/M phase (\%) \\
\hline BEL-7402 & Vehicle & 34.8 & 34.4 & 30.8 \\
& Chidamide $(1 \mu \mathrm{M})$ & 37.2 & 19.4 & 43.4 \\
& Chidamide $(5 \mu \mathrm{M})$ & 42.7 & 15.8 & 41.5 \\
& Chidamide $(10 \mu \mathrm{M})$ & 52.5 & 7.4 & 40.1 \\
& MS-275 $(0.5 \mu \mathrm{M})$ & 33.9 & 18.7 & 47.5 \\
& MS-275 $(2 \mu \mathrm{M})$ & 57.3 & 11.4 & 31.3 \\
HCC-9204 & 61.5 & 6.3 & 32.3 \\
& MS-275 $(5 \mu \mathrm{M})$ & 59.2 & 31.3 & 9.5 \\
& Vehicle & 59.5 & 30.7 & 9.8 \\
& Chidamide $(2 \mu \mathrm{M})$ & 67.0 & 22.6 & 10.5 \\
& Chidamide $(10 \mu \mathrm{M})$ & 69.6 & 16.8 & 13.6 \\
& Chidamide $(20 \mu \mathrm{M})$ & 67.7 & 18.5 & 10.6 \\
& MS-275 $(2 \mu \mathrm{M})$ & 73.9 & 15.5 & 10.8 \\
\hline
\end{tabular}

BEL-7402 and HCC-9204 cells were treated with Chidamide or MS-275 at indicated concentrations for 24 h. Cell cycle of propidium iodidestained cells was analyzed by flow cytometry.

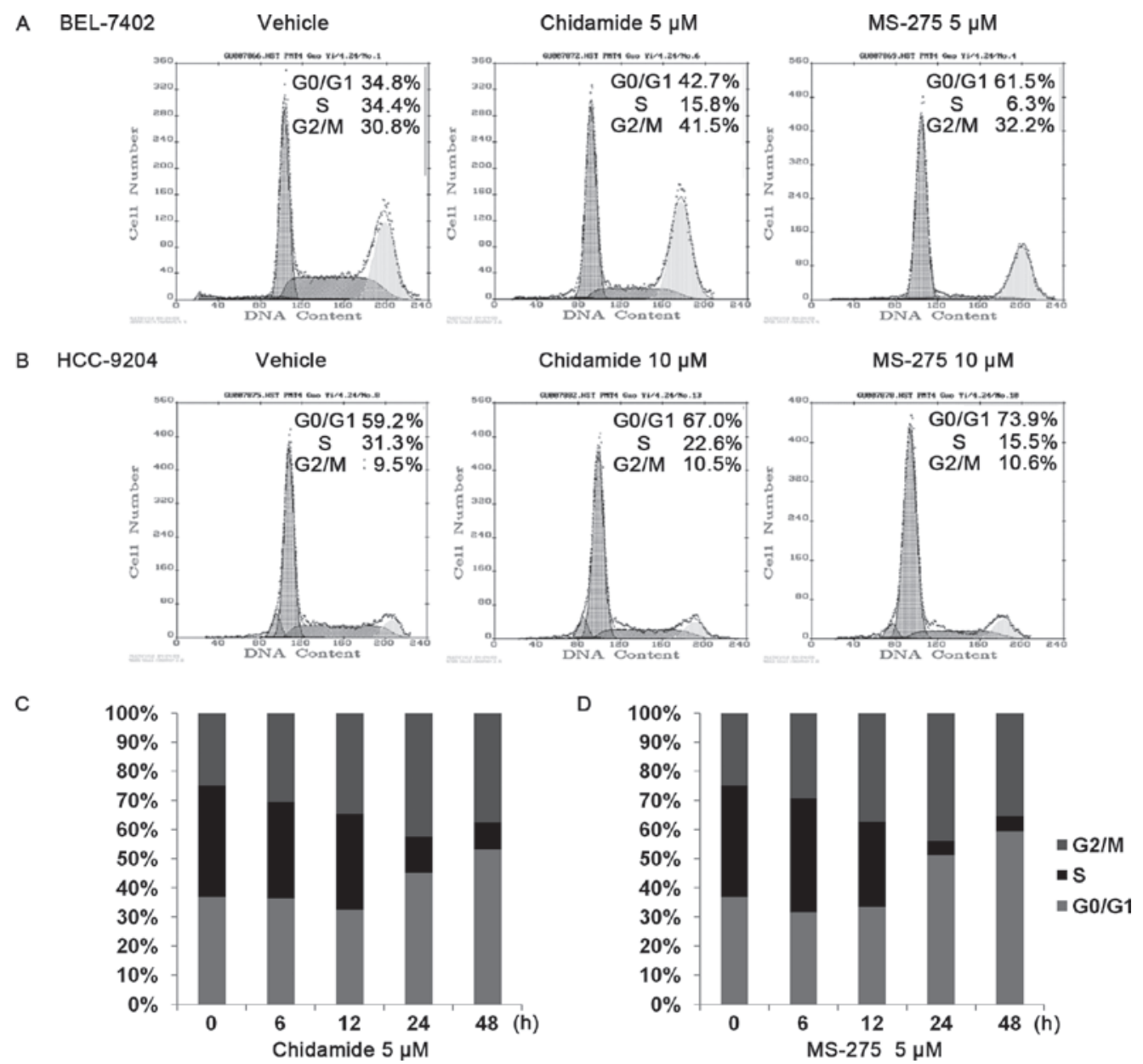

Figure 2. Cell cycle analysis of hepatocellular carcinoma cells after exposure to Chidamide or MS-275 for different times. (A) BEL-7402 and (B) HCC-9204 cells were exposed to $5 \mu \mathrm{M}$ Chidamide or MS-275 (BEL-7402) or $10 \mu \mathrm{M}$ Chidamide or $10 \mu \mathrm{M}$ MS-275 (HCC-9204) for $24 \mathrm{~h}$, and then subjected to flow cytometric analysis of propidium iodide-stained cells. BEL-7402 cells were treated with (C) $5 \mu \mathrm{M}$ Chidamide or (D) $5 \mu \mathrm{M}$ MS-275 for $6,12,24,48 \mathrm{~h}$, after which the percentage of cells in the three phases of the cell cycle was determined by flow cytometric analysis. 
Chidamide $\quad 0$
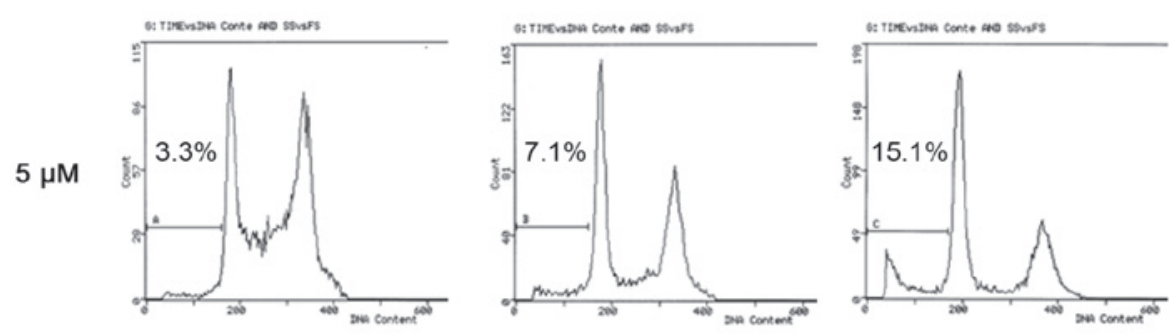

BEL-7402

(h)

$-7402$
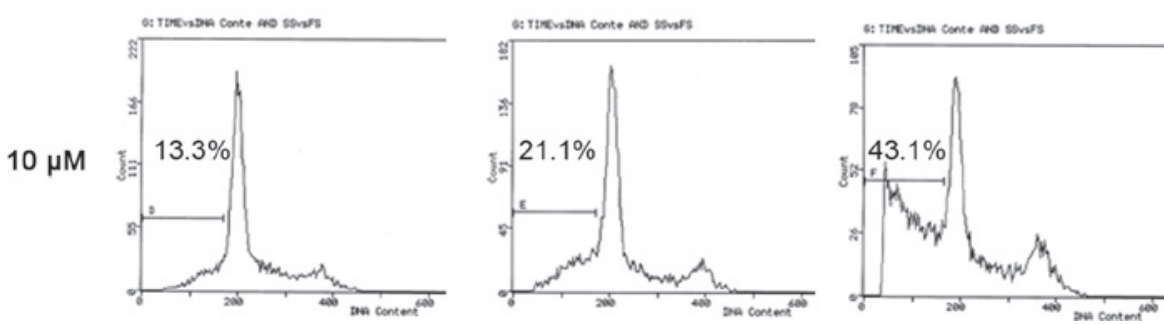

HCC-9204
Figure 3. Apoptosis rate of BEL-7402 and HCC-9204 cells after Chidamide treatment. BEL-7402 and HCC-9204 cells were incubated in either 5 or $10 \mu \mathrm{M}$ Chidamide, respectively, for 24 and $48 \mathrm{~h}$ and then the percentage of apoptotic cells in the sub-G1 phase was analyzed by flow cytometry.

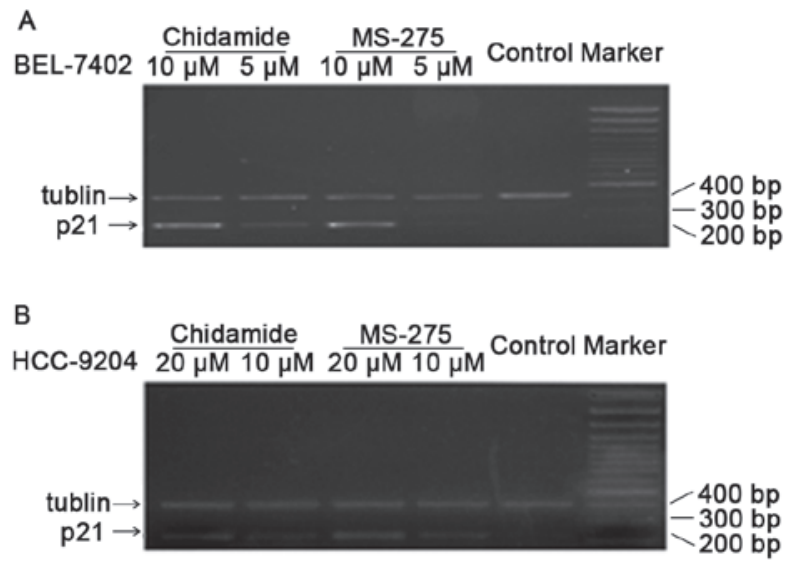

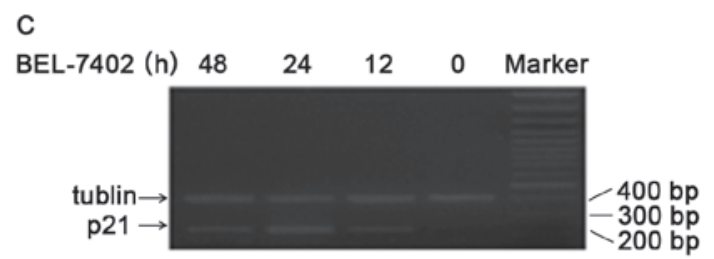

D

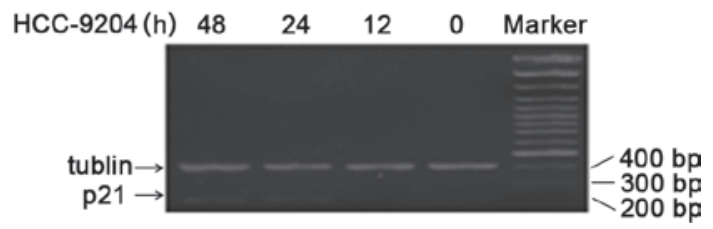

Figure 4. p21 expression induced by Chidamide. (A) BEL-7402 and (B) HCC-9204 cells were exposed to Chidamide or MS-275 at indicated concentrations for $48 \mathrm{~h}$, and then subjected to RT-PCR. (C) BEL7402 and (D) HCC-9204 cells were treated with $5 \mu \mathrm{M}$ (BEL-7402) or $10 \mu \mathrm{M}$ (HCC-9204) Chidamide for 12, 24 or $48 \mathrm{~h}$, after which the expression of p21 mRNA was detected by RT-PCR.

In the present study, the effect of Chidamide on cell growth was evaluated in ten solid tumor cell lines. As shown in Table I, the $\mathrm{IC}_{50}$ of Chidamide and MS-275 were quite similar in half of the studied cell lines. The $\mathrm{IC}_{50}$ of Chidamide was significantly different to that of MS-275 in the other five cell lines, but they were both at $\mu \mathrm{M}$ concentrations. This indicates that Chidamide exhibits broad anticancer effects in vitro, particularly in lung, breast and pancreatic cancer cell lines.

Moreover, to explore whether Chidamide markedly inhibited cell growth, growth curves were plotted for the BEL-7402 and HCC-9204 cells treated with Chidamide. As shown in Table II, Chidamide significantly prolonged the cell doubling time and induced cell death at the dose of $\mathrm{IC}_{50}$. Meanwhile, Chidamide reduced the cell population in the $\mathrm{S}$ phase, during which DNA synthesis occurs, resulting in G1 phase arrest in cancer cells. We also studied the reversibility of cell growth after drug treatment by a growth curve experiment. Cancer cells recovered to normal growth after replacing Chidamide with medium, suggesting that the effect of Chidamide on cell growth inhibition was reversible. Fournel et al demonstrated that HCT116 cell growth resumed after the removal of another MS-275 analog, and acetylated H3 and H4 significantly decreased (11), supporting our data in the present study. In addition, this suggests that the histone acetylation status was closely related to cell growth and viability. Moreover, the combination between HDAC inhibitors and HDAC was a reversible reaction, and HDAC restores activity after removal of inhibitors (12).

Epigenetic modification and chromatin remodeling is the key event during carcinogenesis by upsetting the transcriptional balance between oncogenes and tumor-suppressing genes. The mechanism of the antitumor effect of Chidamide has not yet been clarified. Liu et al showed that Chidamide increased the accumulation of acetylated histone $\mathrm{H} 3$ in colon cancer cells (10). Alternatively, it also increased the acetylation levels of non-histone protein. Herein, we explored the relationship between p21 and Chidamide. It has been known that 
HDAC regulates the acetylation levels of p21 in two ways (13). One way is to change p53 promoter acetylation levels via its deacetylation effect. Therefore, the activity of p53 is suppressed and expression of p21 is reduced. Another is p53-independent, by which HDAC inhibits histone acetylation levels and makes DNA more closely bind to histone. Consequently, transcriptional factors, such as sp1 and E2A, could not combine with binding sites on the promoter region of $\mathrm{p} 21$ which leads to transcription suppression. Notably, the p53-independent pathway is dominant in p21 gene expression up-regulated by HDAC inhibitors $(14,15)$. Our data showed that Chidamide increased p21 mRNA levels in both BEL-7402 cells with wild-type p53 and in HCC-9204 cells with mutant p53 (Fig. 4). In addition, the $\mathrm{IC}_{50}$ between wild-type p53 cell lines and mutant p53 cell lines was not significantly different, which also implies that p21 expression is mainly regulated by an p53-independent pathway.

HDAC inhibitors regulate cell cycle process. For example, MS-275 may decrease the proportion of cells in S phase and increase the proportion of cells in $\mathrm{G} 1$ phase, which causes G1 phase arrest $(16,17)$. Similarly, TSA also induces G1 arrest (18). We evaluated the effect of Chidamide on the cell cycle in BEL-7402 and HCC-9204 cells. Chidamide reduced the percentage of cells in the $\mathrm{S}$ phase and arrested cells at G1 phase, but the change in G2 phase was not consistent, with a slightly increasing trend. MS-275, as a reference component, caused similar results in this study. As a cell cycle-dependent kinase inhibitor, P21 closely integrates with kinases of G1 and $\mathrm{S}$ phase, such as cyclin E/Cdk2, cyclin D/Cdk4 and cyclin A/ Cdk2, blocking transition from G1 to $\mathrm{S}$ phase. Meanwhile, its interation with kinases in $\mathrm{G} 2$ phase is weaker.

Data in the present study suggest that the effect of Chidamide on the cell cycle is dose- and time-dependent. Exposure to Chidamide at less than $\mathrm{IC}_{50}$ dose and less than $24 \mathrm{~h}$ did not induce a significant change in cell cycle of BEL-7402 cells (Fig. 2C). Notably, p21 mRNA levels displayed a similar trend evaluated by RT-PCR. This again indicates that p21 is closely related to the cell cycle arrest induced by Chidamide. However, P21 is not an exclusive factor involved in cell cycle arrest resulting from Chidamide. Other mechanisms may play a role in this process.

Chidamide induces cell apoptosis, the mechanism of which is not very clear. Our data showed that cell apoptosis began to occur at $24 \mathrm{~h}$ after Chidamide treatment and became more obvious at $48 \mathrm{~h}$, which has the same trend with p21 mRNA expression, except for 12-24 h time lag. It has been known that MS-275-induced apoptosis is largely independent of the extrinsic/receptor-mediated pathway and mainly involves engagement of the intrinsic/mitochondrial-related pathway (19). U937 cells stably expressing ectopic Bcl-2 or Bcl-XL significantly protected cells from MS-275-induced death. Liu et al reported that Chidamide-treated colon cancer cells exhibited swollen mitochondria and increased cleavage of caspase- 3 and PARP (10). It is supposed that the mitochondrial-mediated pathway also plays a critical role in apoptosis caused by Chidamide, but the mechanism requires investigation.

Collectively, the results described indicate that Chidamide is a promising anticancer agent with significant effect on growth inhibition of various cell lines. As an analog of Chidamide, MS-275 selectively inhibits HDAC1 and 9, 2 and 3, but has little effect on HDAC4, 6, 7 and 8 (20). To date, no data on the selec- tivity of Chidamide has been found, and this requires clarified. Meanwhile, to make Chidamide available at the bedside of patients, the anticancer activity of Chidamide alone as well as in combination with chemotherapy drugs in vivo, and the underlying mechanism are the focus of further investigation.

\section{References}

1. Ropero S and Esteller M: The role of histone deacetylases (HDACs) in human cancer. Mol Oncol 1: 19-25, 2007.

2. Walkinshaw DR and Yang XJ: Histone deacetylase inhibitors as novel anticancer therapeutics. Curr Oncol 15: 237-243, 2008.

3. Kelly WK and Marks PA: Drug insight: histone deacetylase inhibitors - development of the new targeted anticancer agent suberoylanilide hydroxamic acid. Nat Clin Pract Oncol 2: 150-157, 2005.

4. Lin HY, Chen CS, Lin SP and Weng JR: Targeting histone deacetylase in cancer therapy. Med Res Rev 26: 397-413, 2006.

5. Adler JT, Hottinger DG, Kunnimalaiyaan $M$ and Chen $H$ : Inhibition of growth in medullary thyroid cancer cells with histone deacetylase inhibitors and lithium chloride. J Surg Res 159: 640-644, 2010.

6. Drummond DC, Noble CO, Kirpotin DB, Guo Z, Scott GK and Benz CC: Clinical development of histone deacetylase inhibitors as anticancer agents. Annu Rev Pharmacol Toxicol 45: 495-528, 2005.

7. Niesen MI and Blanck G: Rescue of major histocompatibilityDR surface expression in retinoblastoma-defective, non-small cell lung carcinoma cells by the MS-275 histone deacetylase inhibitor. Biol Pharm Bull 32: 480-482, 2009.

8. Belien A, De Schepper S, Floren W, et al: Real-time gene expression analysis in human xenografts for evaluation of histone deacetylase inhibitors. Mol Cancer Ther 5: 2317-2323, 2006.

9. Bracker TU, Sommer A, Fichtner I, Faus H, Haendler B and Hess-Stumpp H: Efficacy of MS-275, a selective inhibitor of class I histone deacetylases, in human colon cancer models. Int J Oncol 35: 909-920, 2009.

10. Liu L, Chen B, Qin S, et al: A novel histone deacetylase inhibitor Chidamide induces apoptosis of human colon cancer cells. Biochem Biophys Res Commun 392: 190-195, 2010.

11. Fournel M, Trachy-Bourget MC, Yan PT, et al: Sulfonamide anilides, a novel class of histone deacetylase inhibitors, are antiproliferative against human tumors. Cancer Res 62: 4325-4330, 2002.

12. Catalioto RM, Maggi CA and Giuliani S: Chemically distinct HDAC inhibitors prevent adipose conversion of subcutaneous human white preadipocytes at an early stage of the differentiation program. Exp Cell Res 315: 3267-3280, 2009.

13. Roy S, Packman K, Jeffrey R and Tenniswood M: Histone deacetylase inhibitors differentially stabilize acetylated p53 and induce cell cycle arrest or apoptosis in prostate cancer cells. Cell Death Differ 12: 482-491, 2005.

14. Gui CY, Ngo L, Xu WS, Richon VM and Marks PA: Histone deacetylase (HDAC) inhibitor activation of p21WAF1 involves changes in promoter-associated proteins, including HDAC1. Proc Natl Acad Sci USA 101: 1241-1246, 2004

15. Huang L, Sowa Y, Sakai T and Pardee AB: Activation of the p21WAF1/CIP1 promoter independent of p53 by the histone deacetylase inhibitor suberoylanilide hydroxamic acid (SAHA) through the Sp1 sites. Oncogene 19: 5712-5719, 2000.

16. Altmann A, Eisenhut M, Bauder-Wust U, Markert A, Askoxylakis V, Hess-Stumpp $\mathrm{H}$ and Haberkorn U: Therapy of thyroid carcinoma with the histone deacetylase inhibitor MS-275. Eur J Nucl Med Mol Imaging 37: 2286-2297, 2010.

17. Takai N, Ueda T, Nishida M, Nasu K and Narahara H: Anticancer activity of MS-275, a novel histone deacetylase inhibitor, against human endometrial cancer cells. Anticancer Res 26: 939-945, 2006.

18. Okamoto H, Fujioka Y, Takahashi A, Takahashi T, Taniguchi T, Ishikawa Y and Yokoyama M: Trichostatin A, an inhibitor of histone deacetylase, inhibits smooth muscle cell proliferation via induction of p21(WAF1). J Atheroscler Thromb 13: 183-191, 2006.

19. Rosato RR, Almenara JA and Grant S: The histone deacetylase inhibitor MS-275 promotes differentiation or apoptosis in human leukemia cells through a process regulated by generation of reactive oxygen species and induction of p21CIP1/WAF1. Cancer Res 63: 3637-3645, 2003.

20. Khan N, Jeffers M, Kumar S, et al: Determination of the class and isoform selectivity of small-molecule histone deacetylase inhibitors. Biochem J 409: 581-589, 2008. 